\title{
Acoplamento BRAMS-WW3 para previsão de ondas oceânicas
}

\author{
WW3-BRAMS model coupling for ocean waves forecasting
}

\author{
Renata S. R. Ruiz ${ }^{1}$, Rosio del Pilar Camayo Maita ${ }^{2}$, Haroldo F. de Campos Velho ${ }^{2}$, Saulo R. \\ Freitas $^{2}$, Valdir Innocentini ${ }^{2}$ \\ ${ }^{1}$ Faculdade de Tecnologia (FATEC), São José dos Campos (SP), Brasil \\ ${ }^{2}$ Instituto Nacional de Pesquisas Espaciais (INPE), São José dos Campos (SP), Brasil.
}

\begin{abstract}
Resumo
Uma versão acoplada do modelo de ondas oceânicas WaveWatch III 3.14 (WW3) com o modelo meteorológico de mesoescala BRAMS é apresentada. O modelo WW3 é de uso operacional do CPTEC-INPE para previsão de ondas, bem como o modelo BRAMS, usado para previsão numérica do tempo sobre a América do Sul, com resolução horizontal de $5 \mathrm{~km}$. As condições de contorno para o modelo BRAMS são fornecidas pelo modelo global de circulação atmosférica do CPTEC-INPE. A simulação com o modelo BRAMS para o dia 14/Out/2014 gerou um campo de vento mais próximo do campo de vento observado para a costa do Sudeste brasileiro - dados de observação do satélite Metop-A (sensor ASCAT), quando comparado com o campo de vento gerado pelo modelo GFS. A simulação com o campo de vento gerado pelo BRAMS produz ondas mais altas do que as ondas previstas com o campo de vento do GFS.
\end{abstract}

Palavras-chave: Modelo de ondas WaveWatch III, modelo atmosférico BRAMS, modelo atmosférico GFS, acoplamento de modelos, simulação de mesoescala.

\begin{abstract}
A version of the coupled ocean waves WaveWatch III (WW3) model with mesoscale meteorological model BRAMS is presented. The WW3 is a model for waves forecasting. The BRAMS model is used for numerical weather prediction over South America, with horizontal resolution of $5 \mathrm{~km}$. Both models are executed operationally by the CPTEC-INPE. The boundary conditions for the BRAMS model are provided by the global atmospheric circulation model of the CPTEC-INPE. The simulation with BRAMS for 14/Oct/2014 generated a wind field with better agreement with the observations (satellite Metop- $A, A S C A T$ sensor) to the coast of southeastern Brazil) than the field wind generated by the SFM model. The simulation with wind field generated by BRAMS produces higher waves than ones predicted with GFS wind field.
\end{abstract}

Keywords: Wave model WaveWatch III, atmospheric model BRAMS, GFS atmospheric model, model coupling, mesoscale simulation. 


\section{Introdução}

Previsão de dinâmica de ondas oceânicas é uma informação muito relevante para quaisquer atividades navais. O Centro de Previsão de Tempo e Estudos Climáticos (CPTEC) do Instituto Nacional de Pesquisas Espaciais (INPE) faz a previsão operacional da dinâmica de ondas para a costa do Brasil fazendo uso do modelo WaveWatch III (WW3). A previsão é realizada diariamente no horário 0h GMT. O modelo WW3 é executado sobre 5 domínios: domínio global (resolução espacial de 1x1 grau), 3 domínios da costa brasileira (Sul, Sudeste, Nordeste/Norte, com resolução de $0,25 \times 0,25$ ) e a região que cobre a costa do estado do Rio de Janeiro (resolução de $0,05 \times 0,05)$. O modelo é reinicializado todo dia com a previsão de 24 horas a partir da simulação do dia anterior e não faz uso de rotinas de assimilação de dados.

A forçante do modelo de previsão de ondas é o campo de vento, responsável pela transferência de quantidade de movimento da atmosfera para a superfície do oceano. $\mathrm{O}$ acoplamento oceano-atmosfera é portanto o principal mecanismo para formação e desenvolvimento de ondas. Na verdade, o problema é mais complexo, devido uma dinâmica não linear de interação com fronteiras sólidas (fundo do mar e a costa). Todavia, a representação da forçante do vento é considerada a maior fonte de erros dos modelos de ondas. Esta é a principal motivação para o presente estudo.

Na versão operacional do CPTEC-INPE, o modelo WW3 é executado acoplado ao modelo global GFS (Global Forecast System) - AVN do NCEP/NOAA. O nome AVN é ainda empregado para endereçar os servidores de transferência de dados do modelo GFS. Uma nova versão para o modelo de ondas WW3 foi desenvolvida acoplando o modelo BRAMS, que tem suporte e desenvolvimento pelo CPTEC-INPE, base do modelo ambiental operacional do Brasil CPTEC. Na próxima sessão, é feita uma descrição breve dos modelos de simulação usados nesta pesquisa. Em seguida, os resultados são apresentados em nova sessão. As conclusões e comentários finais estão descritos na última sessão do artigo.

\section{Modelos de Previsão}

As principais características dos três modelos usados na simulação são descritas nesta seção. Os 3 modelos mencionados são: (a) Modelo de ondas oceânicas WaveWatch III; (b) Modelo de circulação atmosférica GFS; (c) Modelo de circulação atmosférica BRAMS.

\subsection{Modelo de Ondas: WaveWatch III}

A nova versão do modelo de previsão de agitação marítima WaveWatch III (versão 3.14), desenvolvido pela
Marine Modeling and Analysis Branch (MMAB) do NCEP Tolman $(1989,2009)$ foi implementada no CPTEC-INPE.

O modelo WW3 difere de seus predecessores WAM (WANDIG, 1988) e WaveWatch II Komen et al. (1994) em vários aspectos, das equações governantes, estrutura do código, métodos numéricos e as parametrizações físicas. A versão WW3 3.14 é estruturada em forma modular, o que permite fácil adaptação de novos métodos numéricos e desenvolvimento de outras/novas parametrizações.

A propagação de ondas é considerada linear e efeitos não lineares importantes são incluídos como termos fontes (ver: "User manual", Seção 2.3 - Tolman (2009), ou ainda, as parametrizações físicas. Os processos físicos incluem crescimento de onda e decaimento devido as ações do vento, interações ressonantes, dissipação, fricção de fundo, surf-breaking e espalhamento devido a interações com o fundo.

O modelo usa uma grade estruturada longitudelatitude (os incrementos em longitude e latitude não precisam ser iguais) ou uma grade Cartesiana (opcional). Os espectros de energia da onda são discretizados usando um incremento constante (cobrindo todas as direções) e uma grade para o número de onda que varia espacialmente.

O modelo é escrito em FORTRAN 90. Pode ser compilado com diretivas OpenMP para máquinas multiprocessadas com memória compartilhada ou com opção de compilação MPI (Message Passing Interface) para computadores multi-processados de memória distribuída. Descrição mais detalhada pode ser obtida nas referências citadas e também no sítio do NCEP.

\subsection{Modelo Atmosférico GFS}

O modelo GFS (Global Forecast System) foi desenvolvido pelo NCEP/NOAA, inicialmente descrito por Sela (1980). É um modelo spectral 3D com transformação para uma grade gaussiana para os cálculos de termos não lineares e processos físicos. A coordenada sigma é adotada para a direção vertical, que é definida da superfície (sigma=1) até o topo da atmosfera (sigma=0). O domínio vertical é dividido em camadas, com resolução mais fina próximo à superfície.

O principal laço de integração temporal é realizado com um esquema leapfrog para os termos de advecção não lineares e um esquema semi-implícito para ondas de gravidade e para a advecção zonal da vorticidade e umidade. A dinâmica e a física são executadas em separado. A física está escrita de forma incremental e é executada sequencialmente.

As equações da dinâmica incluem vorticidade, divergência, logaritmo da pressão de surface, umidade, temperatura virtual e condensado de nuvem. Parametrizações para onda de gravidade, radiação, convecção, 
convecção rasa, fração de nuvens, condensação e precipitação em escala de grade e física da camada limite estão presentes e são os módulos mais alterados e/ou atualizados do modelo (Environmental Modeling Center, 2003). O desempenho do modelo tem sido avaliado por inúmeros testes, sob diferentes condições, Yang et al. (2006) realizaram avaliação do modelo e registraram seus resultados.

É um modelo adequado para simulação da dinâmca da atmosfera e disponível para download em:GFS.

\subsection{Modelo Atmosférico BRAMS}

O modelo BRAMS (Freitas et al., 2009) recebe suporte e desenvolvimento pelo CPTEC-INPE, e está baseado no modelo RAMS (Regional Atmospheric Modeling System) (Walko et al., 2000), com novas funcionalidades e parametrizações, simulando vários processos atmosféricos em várias escalas.

Para verificar a instalação foi realizado um teste com o modelo sobre a região global. Os resultados obtidos foram comparados com os resultados obtidos pelo WW3 operacional do CPTEC.

O modelo BRAMS é codificado em diferenças finitas, com utilização de grades telescópicas. A grade vertical é mais refinada próxima ao solo. A integração no tempo usa o esquema Leapfrog. Possui versão hidrostática e nãohidrostática e diversos tipos de parametrizações para turbulência (7 tipos), condensação ( 2 opções), microfísica de nuvens (4) e radiação (5).

O modelo de superfície é o LEAF (Land Ecosystem Atmosphere Feedback) (Lee (1992),Lee e Pielke (1992)). A versão LEAF-2 inclui cobertura vegetal, solo, lagos, oceanos, cobertura de neve e a influência de cada componente na interação com a atmosfera.

\section{Experimentos Numéricos}

As etapas da pesquisa compreenderam a instalação e avaliação dos modelos. Para verificar a instalação foi realizado um teste com o modelo sobre a região global. Os resultados obtidos foram comparados com os resultados obtidos pelo WW3 operacional do CPTEC-INPE e foram considerados satisfatórios.

Os domínios espaciais para o BRAMS são os mesmos da versão operacional, mas algumas adaptações foram necessárias. Deste modo, foram feitas adaptações nos scripts de execução incorporando novos limites de latitude e longitude. Houve interação com o grupo de operação do CPTEC-INPE, que realizou modificações nos scripts do BRAMS para viabilizar a escrita dos arquivos do campo de vento, sem causar, um aumento significativo no tempo de execução. A Figura 1 apresenta o novo subdomínio definido para a região sudeste.

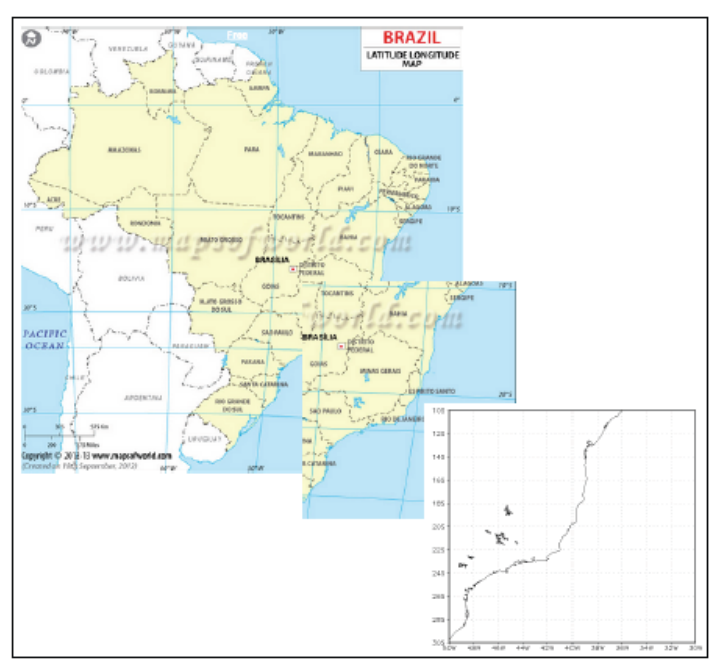

Figura 1: Domínio de integração espacial para os modelos WW3-BRAMS: 10S-30S x 50W-30W.

Para a instalação e compilação do modelo BRAMS são necessários compiladores $C$ e Fortran (intel: icc, ifort ou GNU: g95 e gcc), biblioteca MPI (Messege Passing Interface) e biblioteca HDF (Hierarchical Data Format). O modelo BRAMS versão 5.0 foi instalado e configurado no supercomputador Cray XE6 do CPTEC-INPE. O campo de vento gerado pelos modelos BRAMS e GFS estão mostrados na Figura 2 e Figura 3, respectivamente.

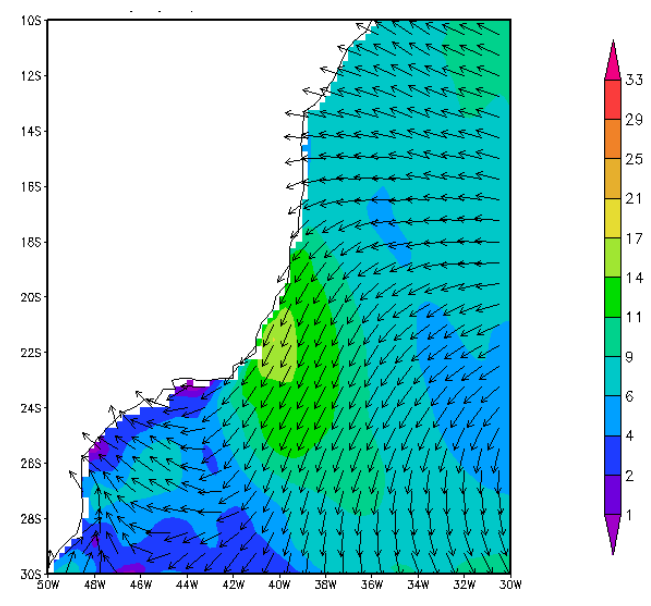

Figura 2: Campo de vento BRAMS

Se observamos, por exemplo, a região onde a intensidade do vento do modelo BRAMS registra 11 e 14 $\mathrm{m} / \mathrm{s}$ (aproximadamente entre 40 e $50 \mathrm{~km} /$ hora), a região abrange uma área com maior extensão ao sul $(\approx 26 S)$ do que as observadas no modelo GFS, que ficaram mais restritas ao norte de 24S. Essas diferenças observadas nos ventos podem influenciar diretamente o campo de ondas simulados pelo modelo WW3. Como já mencionado, a maior fonte de erros dos modelos de ondas é atribuída à representação do acoplamento com o campo 

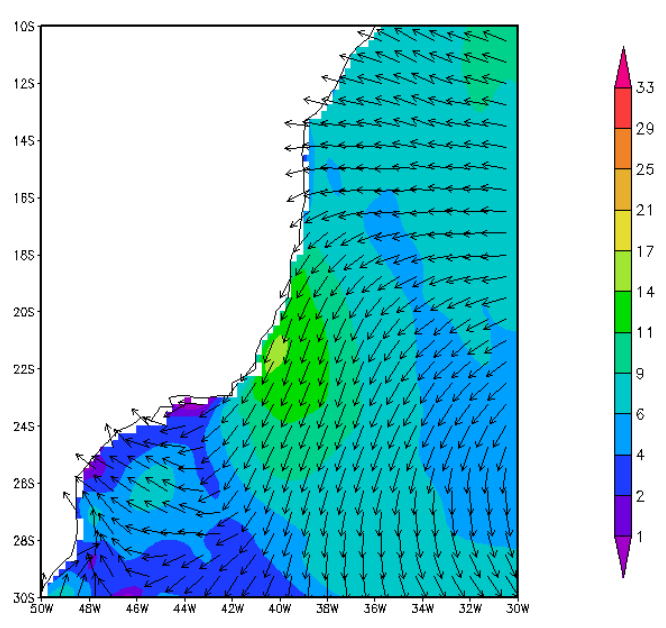

Figura 3: Campo de vento GFS

de vento. De fato, o campo de ondas Figura 4 e Figura 5 mostra uma diferença entre os resultados obtidos com WW3+BRAMS e WW3+GFS.


Figura 4: Campo de ondas WW3+BRAMS

No campo de ondas gerado com WW3-BRAMS, notase um núcleo com ondas mais altas (acima de 2,7 metros) em torno de 26S/40W, o que deve estar associado aos ventos mais intensos na região. Finalmente, a Figura 6 mostra o campo de vento com dados estimados por satélite etop-A (sensor ASCAT).

\section{Conclusões e Comentários Finais}

No campo forçado com os ventos do GFS, as ondas não superam alturas de 2,7 m. As diferenças encontradas deixam indícios de uma melhor representação dos ventos pelo modelo atmosférico de maior resolução, nesse caso o modelo BRAMS, comparado com modelos de baixa resolução como o modelo GFS. Comparando os campos espaciais da intensidade dos ventos para

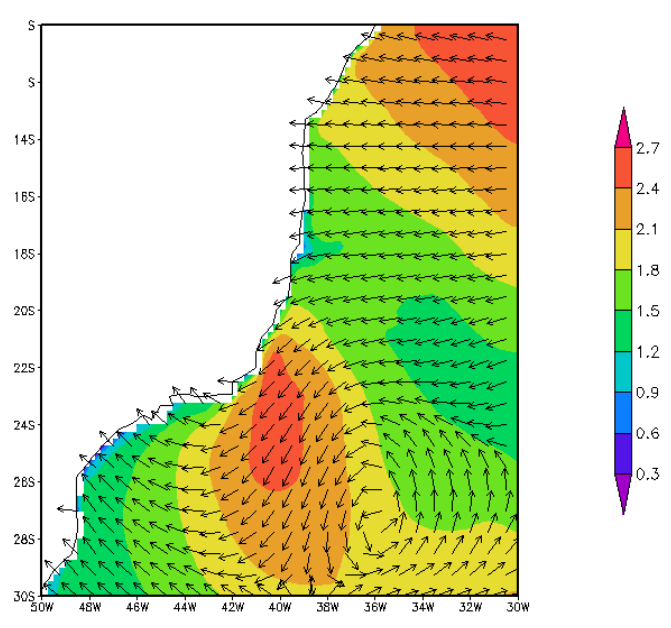

Figura 5: Campo de ondas WW3+GFS

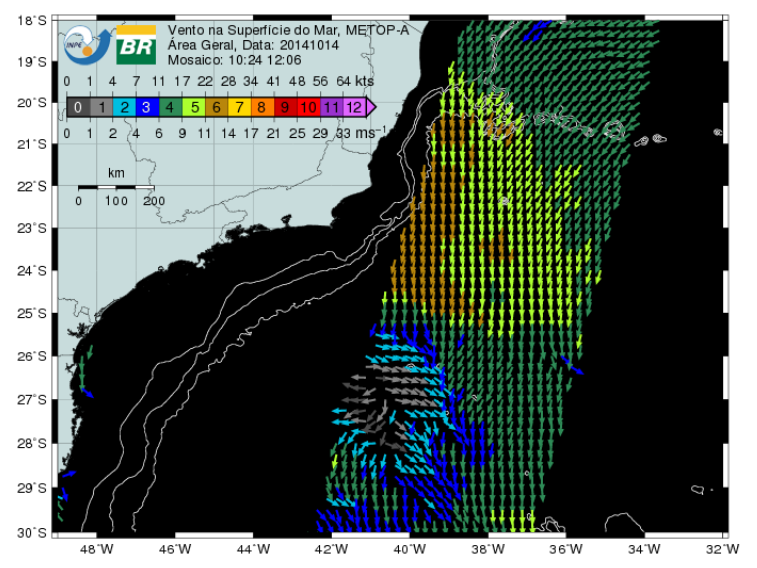

Figura 6: Campo de vento obtido via satélite.

o dia 14/Out/2014 simulados com o modelo BRAMS (Figura 2) e GFS (Figura 3) com dados estimados por satélite etop-A (sensor ASCAT) - ver Figura 6, destaca-se uma diferença na região dos ventos máximos entre 195 e 25.5S (aproximadamente). Os ventos mais intensos cobrem uma maior região no modelo BRAMS que no modelo GFS, indicando uma melhor concordância dos ventos simulados pelo BRAMS com os dados do satélite. Mais estudos sobre o desempenho dos modelos atmosféricos e de ondas deverão ser realizados para um aprofundamento no assunto.

\section{Agradecimentos}

Os autores agradecem ao CNPq e a FAPESP (agências brasileiras de suporte à pesquisa científica) pelo apoio financeiro. 


\section{Referências}

Environmental Modeling Center (2003). The GFS Atmospheric Model. NCEP Office Note 442, Global Climate and Weather Modeling Branch, Maryland (USA).

Freitas, S. R., Longo, K. M., Silva Dias, M. A. F., Chatfield, R., Silva Dias, P. L., Andreae, M. O., Grell, G., Rodrigues, L. F., Fazenda, A., Panetta, J. (2009). The coupled aerosol and tracer transport model to the brazilian developments on the regional atmospheric modeling system (catt-brams) part 1: Model description and evaluation. Atmos Chem Phys, 9, 2843-2861.

Komen, G. J., Cavaleri, L., Donelan, M., Hasselmann, K., Hasselmann, S., Janssen, P. A. E. M. (1994). Dynamics and Modelling of Ocean Waves, $2^{\circ}$ edn. Cambridge University Press..

Lee, T. J. (1992). The impact of vegetation on the atmospheric boundary layer and convective storms. Tese de Doutorado, Colorado State University, Fort Collins, Fort Collins, CO.

Lee, T. J., Pielke, A., R. (1992). Estimating the soil surface specific humidity. Journal of Applied Meteorology, 31, 480-484.

Sela, J. (1980). Spectral modeling at the national meteorological center. Monthly Weather Review, 108, 1279-1292.

Tolman, H. L. (1989). The numerical model wavewatch: a third generation model for the hindcasting of wind waves on tides in shelf seas. Communications on $\mathrm{Hy}$ draulic and Geotechnical Engineering, 89(2).

Tolman, H. L. (2009). User manual and system documentation of WAVEWATCH III version 3.14. NOAA / NWS / NCEP / MMAB Technical Note, descobrir.

Walko, R., Band, L., Baron, J., Kittel, F., Lammers, R., Lee, T., Ojima, D., Pielke, R., Taylor, C., Tague, C., Tremback, C., Vidale, P. (2000). Coupled atmospherebiophysics-hydrology models for environmental modeling. Journal of Applied Meteorology, 39(6), 931-944.

WANDIG (1988). The WAM model - a third generation ocean wave prediction model. Journal of Physical Oceanography, 18, 1775-1810.

Yang, F., Pan, H. L., Krueger, S., Moorthi, S., Lord, S. (2006). Evaluation of the NCEP global forecast system at the ARM SGP site. Monthly Weather Review, 134(12), 3668-3690. 